\title{
Several wall-associated kinases participate positively and negatively in basal defense against rice blast fungus
}

\author{
Amandine Delteil ${ }^{1,2}$, Enrico Gobbato ${ }^{2}$, Bastien Cayrol ${ }^{2}$, Joan Estevan², Corinne Michel-Romiti ${ }^{2}$, Anne Dievart ${ }^{3}$, \\ Thomas Kroj ${ }^{2}$ and J.-B. Morel ${ }^{2^{*}}$
}

\begin{abstract}
Background: Receptor-like kinases are well-known to play key roles in disease resistance. Among them, the Wall-associated kinases (WAKs) have been shown to be positive regulators of fungal disease resistance in several plant species. WAK genes are often transcriptionally regulated during infection but the pathways involved in this regulation are not known. In rice, the OsWAK gene family is significantly amplified compared to Arabidopsis. The possibility that several WAKs participate in different ways to basal defense has not been addressed. Moreover, the direct requirement of rice OSWAK genes in regulating defense has not been explored.

Results: Here we show using rice (Oryza sativa) loss-of-function mutants of four selected OsWAK genes, that individual OsWAKs are required for quantitative resistance to the rice blast fungus, Magnaporthe oryzae. While OsWAK14, OsWAK91 and OsWAK92 positively regulate quantitative resistance, OsWAK112d is a negative regulator of blast resistance. In addition, we show that the very early transcriptional regulation of the rice OsWAK genes is triggered by chitin and is partially under the control of the chitin receptor CEBiP. Finally, we show that OsWAK91 is required for $\mathrm{H}_{2} \mathrm{O}_{2}$ production and sufficient to enhance defense gene expression during infection.

Conclusions: We conclude that the rice OsWAK genes studied are part of basal defense response, potentially mediated by chitin from fungal cell walls. This work also shows that some OsWAKs, like OsWAK112d, may act as negative regulators of disease resistance.
\end{abstract}

Keywords: Rice, Wall-associated kinase (WAK), Basal immunity, Blast fungus

\section{Background}

Plants have evolved the ability to detect potentially pathogenic microorganisms via pattern-recognition receptors (PRRs) localized on the surface of plant cells [1]. PRR proteins recognize Pathogen Associated Molecular Patterns (PAMPs) that are conserved motifs in the pathogen and Damage Associated Molecular Patterns (DAMPs) that derive from the damages caused by pathogen ingress [2]. Detection of pathogen through PRRs triggers PAMPtriggered immunity (PTI, also called basal defense) which is accompanied with rapid production of reactive oxygen species (ROS), activation of mitogen-activated protein

\footnotetext{
*Correspondence: jbmorel@cirad.fr

${ }^{2}$ INRA, UMR BGPI INRA/CIRAD/SupAgro, Campus International de Baillarguet,

TA A 54/K, 34398 Montpellier, France

Full list of author information is available at the end of the article
}

kinases (MAPKs) and changes in expression of immunerelated genes [2].

So far eight bacterial, four fungal PAMPs and 20 PRRs have been identified molecularly [3]. The best studied PAMP recognition systems in plants are represented by the bacterial flagellin recognized by the Arabidopsis thaliana FLS2 receptor and the fungal chitin recognized by the CEBiP receptor [1]. The FLS2 protein belongs to the Receptor-like Kinase (RLK) gene family. The typical structure of an RLK is an extracellular receptor domain that recognizes the PAMP molecule, a transmembrane domain and an intracellular kinase domain [4]. The CEBiP protein is composed of an extra-cellular LysM domain anchored to the membrane but does not contain any kinase domain [5]. FLS2 and CEBiP are found associated with RLK proteins like BAK1 in Arabidopsis and 
CERK1 in rice respectively [1]. FLS2 and CERK1 are positive regulators of basal defense since mutations in these genes lead to a decrease of resistance in Arabidopsis [6,7] or to a decrease of basal defense in rice [8]. By contrast to PAMP, our knowledge on DAMP detection is much less advanced and only three pairs of PRRs and DAMP have been identified so far [3]. One of these is the PRR/DAMP pair between the Arabidopsis Wall-Associated Kinase 1 (AtWAK1) and oligogalacturonides (OGs) [9] derived from the pectin embedded in the cell-wall of most plants [10].

Wall-Associated Kinases are characterized by an extracellular domain composed of one or several repeats of the Epidermal Growth Factor (EGF) domain. The EGF domain is known in animals to bind a very large range of small peptides and to dimerize upon calcium binding [11]. EGF- containing proteins can form homo and heterodimers after ligand binding in animals [12]. Based on homology with the kinase domain of five WAKs from Arabidopsis [13], 21 genes coding WAK-like (WAKL) proteins were identified in Arabidopsis and 125 in rice, revealing an expansion of the WAK family in monocots $[14,15]$. For simplicity and following previous nomenclature in rice [15], the WAK-like proteins are referred as WAKs. Among the rice WAKs, 67 have a bona fide EGF extracellular domain. Only a few WAKs from Arabidopsis or rice have been shown to possess kinase activity $[16,17]$. Similarly, only a few WAKs have been localized to the plasma membrane in Arabidopsis [18] or rice (OsWAK1) [17], (OsDEES1/OsWAK91) [19]. More recently, maize ZmWAK was shown to be localized to the plasma membrane [20]. Moreover, WAKs seem to be found in large membrane protein complexes of unknown composition [21]. It is not known whether WAKs associate with other RLKs to ensure appropriate function like several other RLKs [22].

In plants, several ligands were shown to bind the extracellular domain of WAK proteins. For example the AtGRP3 protein binds to AtWAK1 [21] and pectin and OGs bind AtWAK1 and AtWAK2 [23-25]. It was shown that upon pectin treatment AtWAK2 activates the mitogen-activated kinases MPK3 and MPK6 and that a TAP-tagged (Tandem Affinity Purification) version of AtWAK2 constitutively activates ROS production and defense gene expression [26]. However, there is no indication that native WAKs can trigger ROS and there is only very limited information on defense gene expression during infection [20].

WAKs are involved in plant development [27]. For instance, AtWAK1 and AtWAK2 are required for cell wall expansion [28]. Accordingly, WAK mutants are often affected in their development. In rice, plants silenced for OsDEES1/OsWAK91 displayed fertility deficiency [19] that was attributed to a defect in embryo development. Plants silenced for the rice indica OsiWAK1 gene were stunted
[29] and in Arabidopsis, silencing of AtWAK1 and AtWAK2 is lethal [28].

The role of WAKs in plant disease resistance initially came from indirect evidence with WAK mutants affected in the triggering of defense-related response [18]. Later, several studies provided direct evidence that WAK genes participate to resistance. First, it was shown that the RFO1/WAKL22 gene is responsible for quantitative resistance to Fusarium [30] and Verticilium [31]. More recently, two distinct wall-associated kinases from maize were shown to be responsible for a major QTL for resistance to the soil-borne fungus Sporisorium reilianum (ZmWAK) [20] and one against the foliar fungal pathogen Exserohilum turcicum (Htn) [32]. Secondly, several mutant analyses of WAK genes provided evidence for their involvement in disease resistance. The over-expression of AtWAK1 led to enhanced resistance to Botrytis [9] and over-expression of OsWAK1 enhanced resistance to Magnaporthe oryzae [17]. On the other hand, silencing of SlWAK1 in tomato lead to enhanced susceptibility to the bacterial pathogen Pseudomonas synringae pv tomato [33]. Other examples of the effect of WAKs on bacterial and fungal resistance are reported although the corresponding proteins miss an EGF domain (OsWAK25) [34] or a kinase domain (At5g50290) [35]. Thus several WAK mutants seem to act as positive regulators of disease resistance to fungi and bacteria without visible developmental phenotypes. However, there is thus far no indication that PTI is affected in these mutants.

Another indication that WAKs are related to disease response comes from the observation that WAK genes are often regulated by bacterial infection in Arabidopsis [33] and by blast infection in rice [36, 37]. Quite interestingly, there are two cases of pathogens that manipulate WAK gene expression by either expressing small RNA interfering with their RNA [35] or by an unknown mechanism [33]. Thus WAKs are important components of basal defense that pathogens try to inhibit. PAMPs can also directly regulate the expression of WAK genes [38]. Flagellin induces several WAK genes in Arabidopsis [39] and tomato [33]. Chitin induces OsWAK91 in rice in a CEBiP dependent manner in cell cultures [5] and the AtWAKL10 gene in Arabidopsis [40]. However, the global regulation of WAK genes in PTI is not well understood.

Here we report that several rice WAK genes are upregulated while OsWAK112d is down-regulated by fungal infection in rice. Part of this transcriptional control is likely due to chitin detection by the chitin receptor CEBiP. We provide evidence that OsWAK14, OsWAK91 and OsWAK92 act as positive regulators of quantitative resistance, while OsWAK112 acts as a negative regulator. By studying OsWAK91 mutants, we demonstrate that this WAK significantly participates 
to ROS production and defense gene expression during infection.

\section{Results \\ OsWAK expression is influenced by blast infection}

Previous transcriptome analysis identified five OsWAK genes differentially expressed upon infection by $M$. oryzae in rice (Additional file 1). Phylogenetic analysis revealed that excluding OsWAK1, all blast responsive WAKs are from one major clade of rice WAKs designated WAKb and that they belong to four different, clearly distinct WAKb sub clades (Additional file 2).

To further investigate on these blast-responsive WAKs, their expression profile in compatible and incompatible interactions was measured at early and late infection stages ( 1 to $24 \mathrm{~h}$ post-infection (hpi) and 48 to $96 \mathrm{hpi}$ ) using isolates FR13 and CL367 respectively (Fig. 1a). All OsWAKs transcripts were differentially expressed during infection in at least one time point and expression changes were similar in compatible and incompatible interactions. At late infection stages, except OsWAK112d, all OsWAK genes were induced and expression induction was often more evident in susceptible plants than in resistant ones. During early infection (2 and $4 \mathrm{hpi}$ ), the expression of OsWAK90 and OsWAK91 was induced. By contrast, OsWAK112d transcripts, and to a lower extent OsWAK14, were repressed early. Thus, among the various transcriptional changes found for the tested OsWAKs, most were induced during infection, sometimes even before fungal penetration (< 24hpi) and one, OsWAK112d was repressed.

\section{Chitin triggers OsWAK gene expression}

The early and non-isolate specific differential expression of the OsWAK genes (Fig. 1b) suggested that a PAMP common to these isolates was the trigger for OsWAK gene regulation during early infection. Chitin is common to all fungi and has been shown to act as an important PAMP in several biological systems $[7,41]$ including rice $[5,42]$. To test the effect of chitin on OsWAK gene expression, plants were sprayed with chitin oligomers and the expression of OsWAKs was determined. Chitin strongly and rapidly induced the expression of the blast-induced genes (Fig. 1b) OsWAK91 and OsWAK90 (almost 20 fold induction of both genes after $1 \mathrm{~h}$ ) while the blast-repressed OsWAK112d was down-regulated (8-fold) by the chitin treatment. The expression of OsWAK14 and OsWAK92 was induced to a much lower extent by the chitin treatment. Therefore, we conclude that OsWAK genes show similar expression trends after chitin treatment (Fig. 1b) and during early stages of blast infection (Fig. 1a).

In order to test whether chitin regulates OsWAKs in a receptor-dependent manner, OsWAK gene expression was analyzed in mutant lines deficient for CEBiP, the major chitin receptor in rice $[5,42,43]$. Chitin oligomers were sprayed on cebip loss-of-function mutant plants [44] and gene expression was measured until $2 \mathrm{~h}$ after treatment (Fig. 1c). Mutants in CEBiP have been shown to display a reduced transcriptional response of OsWAKs to chitin oligomers [43]. For OsWAK90 and OsWAK91, chitintriggered gene expression was significantly reduced in the cebip mutant (Fig. 1c). By contrast the induction of the other chitin-responsive OsWAK genes and the repression of OsWAK112d were only slightly affected by cebip mutation. This supports our hypothesis that the CEBiP receptor is required for proper activation and repression of several OsWAK genes upon chitin treatment.

\section{Different requirements of OsWAKs for quantitative resistance to rice blast}

To elucidate the role of blast- and -chitin responsive WAKs in disease resistance, mutant lines were searched in the OryzaTagLine mutant collection [45, 46]. Two allelic lines for OsWAK14 (wak14-1 and wak14-2) and one line for each OsWAK91, OsWAK92 and OsWAK112d were identified (Additional file 3A). The insertion lines harboured a Tos 17 retrotransposon inserted into the coding sequence of the respective OsWAK genes. For each insertion line, we isolated one homozygous line for the Tos 17 element (mutant) and one sister line without the Tos17 element (later called null-segregant: NS). We confirmed that the expression of the targeted OsWAK gene was reduced in each mutant line as compared to the nullsegregant line (Additional file 3B). The mutant lines did not show any obvious growth phenotype (data not shown), including full fertility in the wak91 mutant despite previous report showing that RNAi of this gene leads to sterility [19].

To determine whether the wak mutations could affect $R$ gene mediated resistance, we tested the avirulent $M$. oryzae isolate CL367 on wak mutant and null-segregant lines. After inoculation with isolate CL367, we did not observe any difference between the wak mutants and their respective null-segregant (data not shown).

To test the impact of WAK mutations on basal resistance, we inoculated the wak mutants with the virulent $M$. oryzae isolate FR13. The wak14-1, wak14-2, wak91 and wak92 mutants were all more susceptible to isolate FR13 than their respective null-segregant controls (Fig. 2a, b) and displayed an increased number of sporulating lesions (1.6-fold more for wak14-1, 2.3-fold for wak14-2, 2.5-fold for wak91 and 1.8-fold for wak92). On the opposite, wak112d mutant plants were more resistant to blast disease. This was manifested by a 1.6-fold reduction of disease lesion numbers. Thus wak mutants are affected for blast susceptibility, suggesting that the corresponding OsWAK genes are important elements of basal disease resistance. 


\section{Effects of over-expression of the OsWAK91 and} OsWAK112d genes on basal resistance to blast fungus In order to further investigate the role of the OsWAKs in blast resistance, we decided to produce rice plants that over-express OsWAK91 and OsWAK112d. We focused on these two genes as they represented the most pronounced expression patterns after infection (Fig. 1) as well as the strongest disease phenotypes in the corresponding loss-offunction mutants (Fig. 2).

After infection with the virulent strain FR13, all 10T0 plants over-expressing OsWAK91 showed reduced symptoms compared to plants transformed with the empty vector (Additional file 4A, B). By contrast, over-expression of the OsWAK112d gene increased susceptibility compared

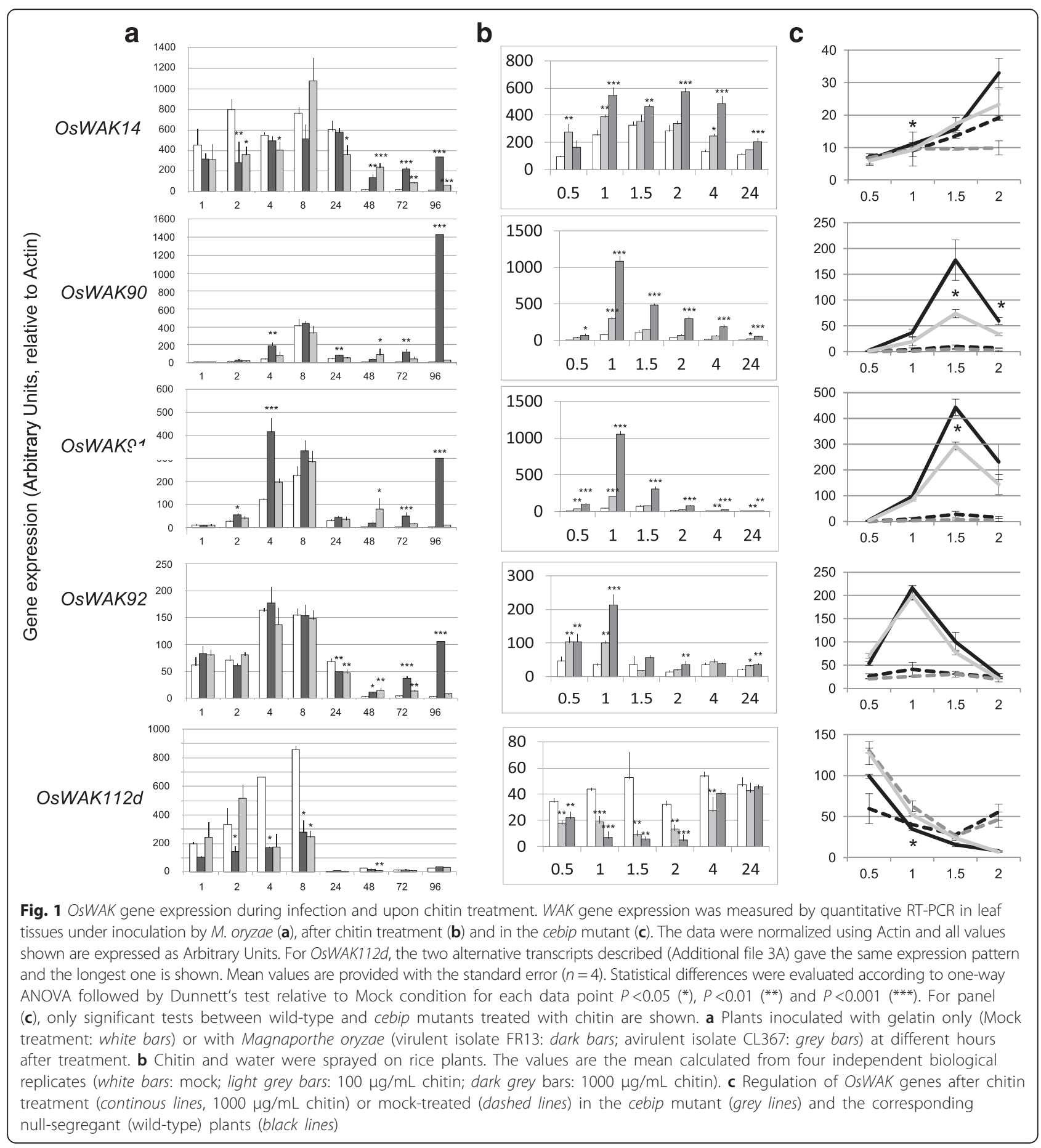




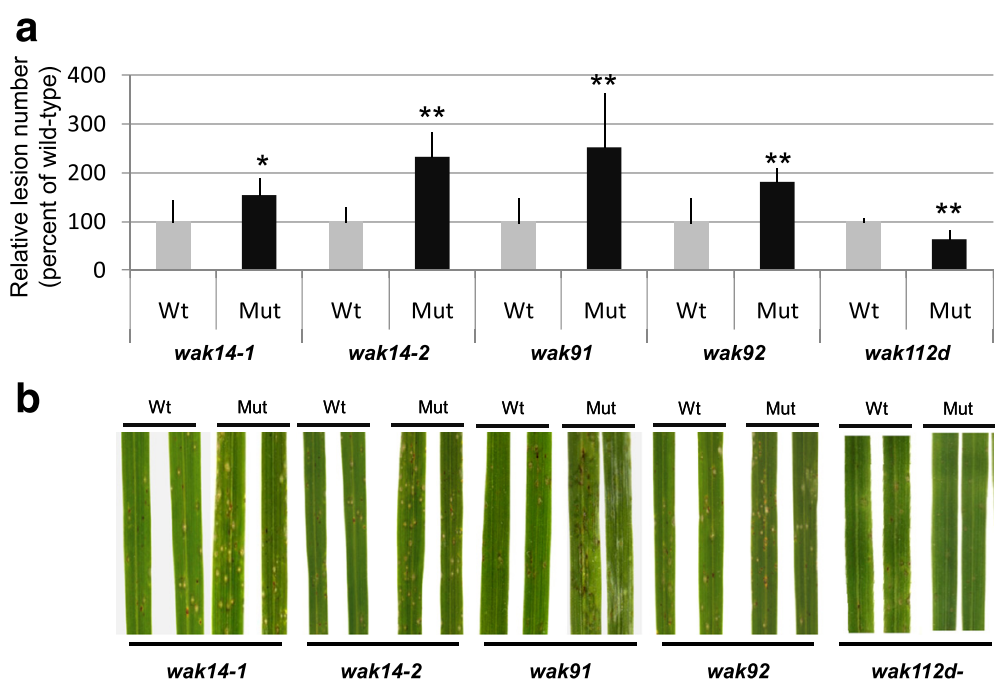

Fig. 2 Resistance to M. oryzae is affected in wak loss-of-function mutants. Plants were inoculated with the virulent isolate FR13 of M. oryzae and disease was measured $7 \mathrm{dpi}$. For each line, a homozygous mutant (Mut) and the corresponding null-segregant (Wt) is presented. a The number of lesions on the youngest fully expanded leaves of at least 7 plants of homozygous and null-segregant lines was counted $7 \mathrm{dpi}$. The values were transformed in percentage relative to the mean of null-segregant lines, and means and standard deviations were calculated. A t-test was performed to establish whether one given mutant line was different from its corresponding null-segregant line $\left(^{*}: p<0.05 ; * *: 0.001\right)$. The experiment was repeated seven times and one representative experiment is shown. $\mathbf{b}$ Pictures were taken 5 days post inoculation. Susceptibility is characterized by the grayish lesions while small brown spots derive from some residual resistance

to empty vector (Additional file 4C, D). In order to further analyze these disease resistance phenotypes, single locus insertion lines were selected. In the T1 generation, plants without T-DNA and sister plants with T-DNA were identified and their seed amplified to give rise respectively to homozygous null-segregants (NS) and over-expresser lines (OE) (Fig. 3a, b). The OE and NS lines were inoculated with the moderately virulent $M$. oryzae isolate GY11. We observed a decrease in lesion number for the OE-WAK91 plants (60\%; Fig. 3c, e) and an increase for the OEWAK112d plants (264\%; Fig. 3d, f). Thus, it appears that the over-expression of OsWAK91 or OsWAK112d alters basal resistance and that both genes have opposite effects on quantitative resistance, consistent with the phenotypes observed with the loss-of-function mutants.

\section{Defense induction in OsWAK91 mutant lines}

To further characterize the increased partial resistance of OE-WAK91 lines, individual interaction sites were examined by microscopy (Additional file 5). Two days after infection, the number of hyphae that penetrated into rice epidermal cells was only slightly reduced in OE-WAK91 lines and 3 days after inoculation, the proportion of fungal hyphae that invaded more than one cell was strongly reduced. These data suggest that OsWAK91 over-expression enhances resistance against fungal penetration and affects the in planta growth of the blast fungus and/or the invasion of new cells.

To get further insights on how OsWAK91 gene affects basal resistance, we measured several molecular markers of basal defense in the OsWAK91 lines (Fig. 4) two days post inoculation, at a time where only small differences in fungal growth were visible (Additional file 5). We first tested whether the oxidative burst, one of the earliest responses to fungal invasion was affected. Two days after inoculation, the production of $\mathrm{H}_{2} \mathrm{O}_{2}$ as measured by DAB staining was two times higher in OE-WAK91 lines and two times lower in ko-WAK91 lines compared to their respective controls (Fig. 4a). Using qRT-PCR on defense marker genes, we then evaluated whether defense was enhanced before and during infection. We could not detect any significant difference of expression of the defense marker genes before inoculation in OEWAK91 compared to control lines (Fig. 4b). By contrast, several markers tested were significantly induced to higher levels in OE-WAK91 plants 48 h post inoculation (Fig. 4b). Thus, we could correlate the increased level of resistance of $\mathrm{OE}-W A K 91$ visible at $3 \mathrm{dpi}$ with an increase in $\mathrm{H}_{2} \mathrm{O}_{2}$ production and defense-gene induction at 2 dpi.

\section{Discussion}

The expression of OsWAK genes is induced by chitin under the control of the CEBiP receptor

Several previous reports indicate that OsWAKs genes are transcriptionnaly regulated during $M$. oryzae infection [36]. By investigating the regulation of OsWAKs during early infection steps, before penetration of $M$. oryzae, in both resistant and susceptible plants we confirmed that five selected OsWAK genes are differentially expressed 


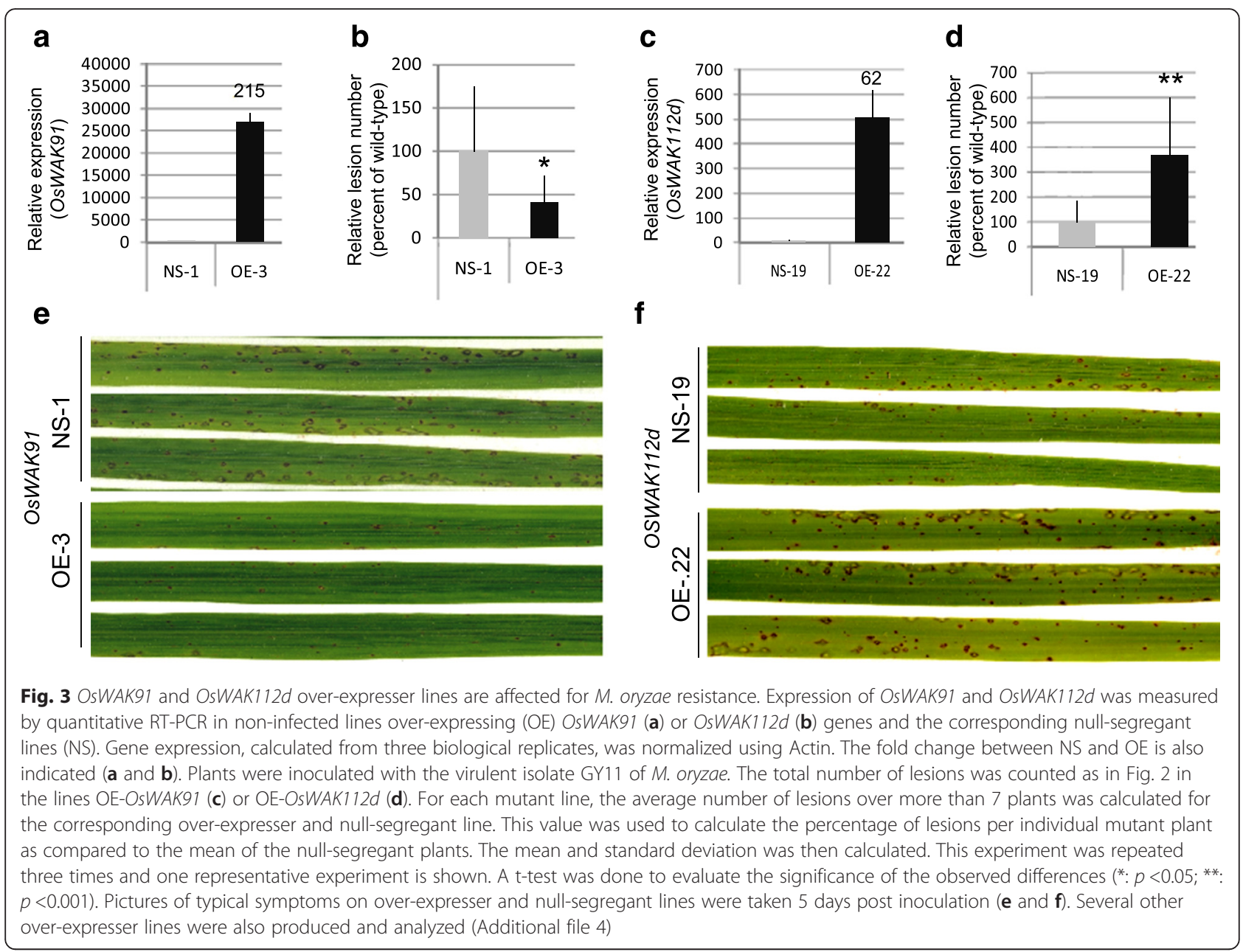

upon infection by virulent and avirulent isolates of $M$. oryzae (Fig. 1a). We showed that the differential expression of the OsWAK genes was, overall, more intense in the case of quantitative resistance (compatible interaction with FR13 isolate) than in complete resistance (incompatible interaction with CL367 isolate). This is contrasting with the report that the OsWAK1 gene is more induced in resistant plants than in susceptible plants [17]. Quite interestingly, the OsWAK1gene belongs to another phylogenetic group than all OsWAKs tested here (Additional file 2). This may explain these differences in transcriptional regulation.

The OsWAK91 and OsWAK112d genes were characterized by their differential expression early after inoculation ( $2 \mathrm{hpi}$, before penetration). This is also contrasting with the observation that the OsWAK1 gene is induced rather late after infection (16-24 hpi) [17]. Quite notably, the OsWAK112d gene was downregulated in the early phases of infection (Fig. 1a) and as expected from Vergne et al. [37] was slightly induced after $24 \mathrm{hpi}$. The triggering of some of OsWAKs expression before fungal penetration $(<24 \mathrm{hpi})$ suggested that a molecule constitutively present in fungi could elicit this expression. Chitin, a molecule that is known to elicit defenses in rice [5] was tested. Chitin alone triggered induction of the OsWAK9O and OsWAK91 genes and the repression of the OsWAK112d gene (Fig. 1b), thus mimicking the events observed during infection (Fig. 1a). Using the cebip mutant, we show that the regulation of expression by chitin of OsWAK90 and OsWAK91, and to a lower extent the other OsWAK tested, is controlled by CEBiP. Thus OsWAK gene regulation by chitin is partially affected by the cebip mutation. Testing CERK1 [8], LYP4 and LYP6 [47] mutants is required to establish if these other chitin receptors are involved in triggering OsWAKs expression. AtWAK1 and $A t W A K 2$ genes are slightly down-regulated by flg22 $[9,38]$ while AtWAKL genes are up-regulated [39]. Similarly, transcriptome analysis indicates that the expression of the AtWAKL10 gene is triggered by chitin [40]. Thus WAK gene regulation by PAMPs seems to be a common feature in plants. 


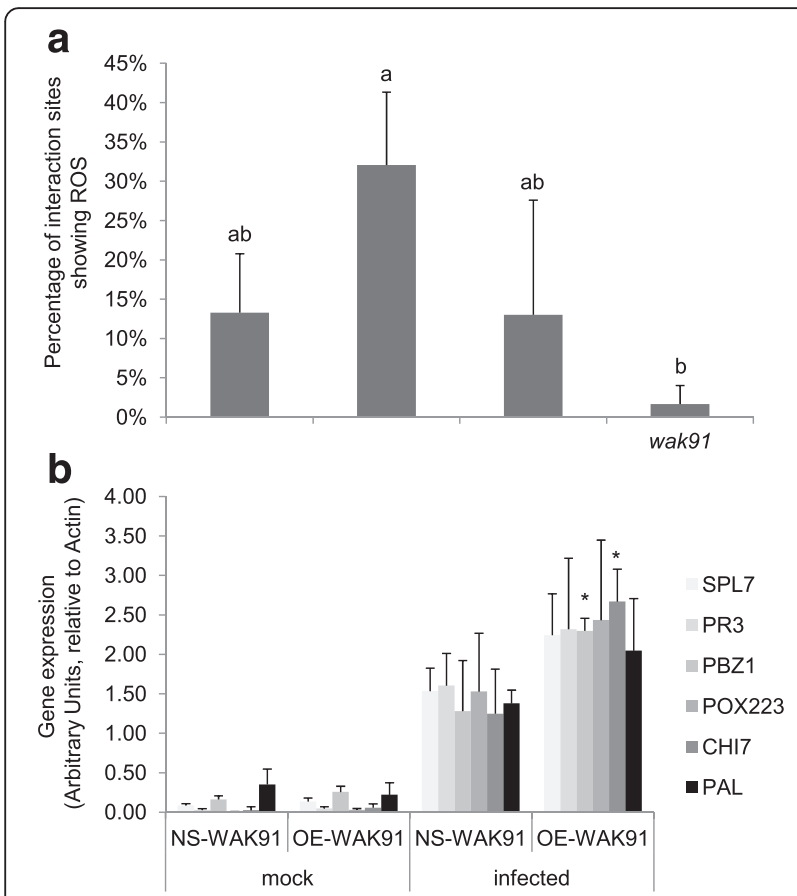

Fig. 4 Defense gene expression and $\mathrm{H}_{2} \mathrm{O}_{2}$ production in OE-WAK91 lines. OE-WAK91 lines were inoculated with M. oryzae (GY11 moderately virulent isolate) and we measured two facets of plant defense response at 48 hpi: $\mathrm{H}_{2} \mathrm{O}_{2}$ production (a) and expression of genes responsive to infection (b). Mean values are provided with the standard error $(n=4)$. Statistical differences were evaluated according to one-way ANOVA followed by Fischer's (a) or Dunnett's (b) tests: $P<0.05\left({ }^{*}\right), P<0.01\left(^{* *}\right)$ and $\left.P<0.001{ }^{(* *}\right)$. a $\mathrm{H}_{2} \mathrm{O}_{2}$ production in loss-of function and $\mathrm{OE}-$ OsWAK91 was expressed as the percentage of infection sites $>100$ counted for each genotype) showing DAB staining. $\mathbf{b}$ Expression of defense-related genes in the OsWAK91 over-expresser (OE) and null-segregant (NS) lines

\section{OsWAK genes are required for quantitative resistance to rice blast fungus}

There are now six reports that WAK genes are involved in disease resistance. In Dicots, several studies report on the role of WAKs in disease resistance: RFO1/WAKL22 [30, 31], AtWAK1 [9], SlWAK1 [33]. In rice, there is only one report that the over-expression of the OsWAK1 gene enhanced resistance to $M$. oryzae [17]. More recently two QTL for resistance to fungal disease in maize were shown to encode $W A K$ genes [20,32]. Our work showing the involvement of four OsWAK genes in rice blast resistance significantly extends this list and reinforces the notion that these receptor-like kinases play a central role in basal resistance. Indeed, we show that three lossof-function mutants in OsWAK14, OsWAK91 and OsWAK92 have reduced basal resistance towards a virulent isolate of the rice blast fungus (Fig. 2). The two independent mutant alleles of the OsWAK14 gene displayed similar phenotypes, suggesting, despite the lack of complementation analysis, that OsWAK14 is a positive regulator of blast resistance. The fact that OsWAK91 loss-of-function displayed reduced resistance combined to the fact that over-expression of the OsWAK91 gene leads to enhanced resistance (Fig. 3a, c, e) suggests that this gene is also a positive regulator of blast resistance. Whether OsWAK92 is also a positive regulator of basal resistance awaits additional genetic demonstration.

Quite strikingly, the mutant in the OsWAK112d gene and over-expression of the OsWAK112d gene led to increased resistance and susceptibility respectively. Altogether with the observation that this gene is repressed upon infection (Fig. 1a), this indicates that OsWAK112d is a negative regulator of basal resistance that rice plants repress during infection. Similarly, the Arabidopsis LRR-RLK FERONIA gene was shown to negatively regulate the signaling pathway involved in basal defense [48]. Thus our results extend the observation that negative regulation by RLK of signaling pathways could be a common trend in plants.

Thus far the kinase domains of the majority of RLK involved in disease resistance belong non-RD group [4], including OsWAK1. It is noteworthy that all OsWAKs tested in this study belong to the WAKb sub-group (Additional file 2) which are characterized by kinase domains of the RD type [49], like all known Arabidopsis WAKs. Thus this work indicates that several OsWAK genes from this WAKb/RD kinase sub-group are also regulators of quantitative resistance to the blast fungus in rice.

\section{OsWAK91 participates in plant defense response}

Measurement of $\mathrm{H}_{2} \mathrm{O}_{2}$ production (Fig. 4a) and defense genes expression (Fig. 4b) in OsWAK91 mutant lines indicated that this gene is involved in both responses to pathogen. This is consistent with the enhanced basal resistance levels observed in over-expressing plants (Fig. 3a, c, e). In particular plants over-expressing OsWAK91 displayed enhanced $\mathrm{H}_{2} \mathrm{O}_{2}$ production and defense-related gene expression. Only limited evidence suggests that WAKs can regulate, directly or indirectly, defense-gene expression. Indeed, in their study, Zuo et al. [20] provided some evidence that three genes (NPR1, PR5 and LOX1) out of six tested were slightly upregulated by ZmWAK upon infection. Our results are consistent with such slight modifications. By contrast to Zuo et al. [20], we did not observe any enhanced expression of defense-marker genes before inoculation triggered by the over-expression of OsWAK91. Thus WAKs may have different ways for activating defense.

As proposed by others [33], our data support a model in which recognition of PAMPs like chitin would lead to an increased expression of OsWAKs and an increase in OsWAK receptors at the plasma membrane. Subsequently, DAMPs produced by pathogen ingress could be recognized by OsWAK91, triggering an enhanced immune response. 
Further experiments are needed to test this model, such as extensive time course analysis of defense response in wak91 mutants.

\section{Conclusions}

We have shown that several OsWAK genes are required for quantitative resistance in rice to the blast fungus $M$. oryzae. More importantly, we showed that among the four OsWAK genes functionally analyzed, one is a negative regulator. In Arabidopsis, pectin was shown to bind AtWAK1 and AtWAK2 and to activate signal transduction [25]. Whether pectins play a similar role in rice needs to be tested. More generally, identifying the molecular signal(s) recognized by these OsWAKs proteins is now possible by the use of the mutants lines described in this study.

\section{Methods}

\section{Identification of the wak mutants}

Insertion mutants and corresponding null-segregant mutants (wild-type) were identified for the OsWAK genes in the OryzaTagLine mutant collection in the Nipponbare background [45, 46]. For each insertion line, PCR was used to select null mutants (siblings from homozygous line) and homozygous mutant plants in a segregating T2 family. The primers used are given in Additional file 6 . These plants were allowed to self and the genotypes were confirmed by PCR in the T3 generation.

\section{Production of over-expresser plants}

The full lengh cDNA of OsWAK91 and OsWAK112d were amplified by RT-PCR using cDNA obtained from a mixture of infected and non-infected Nipponbare plants (primers in Additional file 6). The PCR products were ligated in the pCAMBIA $2300 \mathrm{OX}$ (kindly provided by JC Breitler, CIRAD) vector under the control of the ubiquitin promoter, using the BP reaction (Gateway). The inserts of the corresponding vectors were sequenced before transformation into Agrobacterium tumefaciens (strain EHA105). The resulting pUbi::WAK constructs were transformed into the rice embryo callus of Nipponbare, and transgenic plants were selected on plates containing $200 \mathrm{mg} / \mathrm{l}$ geneticin, $400 \mathrm{mg} / \mathrm{l}$ cefotaxine and $100 \mathrm{mg} / \mathrm{l}$ vancomycine. The primary transformants (T0 plants) were transplanted into soil and allowed to self. DNA from T0 plants was extracted for Southern blot hybridization to screen for single insertion lines. The resulting $\mathrm{T} 1$ single insertion lines were screened by PCR for the presence/absence of the selection marker to identify null-segregants and siblings presenting the T-DNA. These T1 plants were allowed to self and the resulting homozygous $\mathrm{T} 2$ seedlings were used for phenotyping upon $M$. oryzae infection.
Fungal isolates, infection assays and chitin treatment Rice plants and fungi were grown as described in Berruyer et al. [50] under $12 \mathrm{~h}$ light with temperatures between 28 and $30{ }^{\circ} \mathrm{C}$ One rice cultivar, Nipponbare (Oryza sativa L.) and three isolates, FR13, GY11 and CL367 of blast fungus (Magnaporthe oryzae) were used. The isolate CL367 is incompatible and isolates FR13 and GY11 are compatible with Nipponbare [44]. For disease phenotyping, one typical replicate was made of 4 mutant and 4 null-segregant plants grown side-by-side in $1 \mathrm{~L}$ pots; inoculation was carried out by spraying $25 \times 10^{3}$ conidia/mL of FR13 or GY11 isolates of $M$. oryzae (compatible strains) whereas for expression analyses we used $2 \times 10^{5}$ conidia/mL of FR13 and CL367 conidial suspension, on fourth leaves (last fully expanded) from the bottom of 4 week old plants. All treated seedlings were placed in the dark with $100 \%$ relative humidity for $24 \mathrm{~h}$ and at $20-24{ }^{\circ} \mathrm{C}$. For mutant phenotyping, the fourth leaves were harvested and scanned at 5 days after infection for lesion observation and quantification. We differentiated susceptible lesions from resistant lesions by the presence or absence of grayish centers, indicative of sporulation.

For chitin treatment, 3 week-old Nipponbare plants (showing three fully expanded leaves) were sprayed with $0.02 \%$ tween 20 (mock), $100 \mu \mathrm{g} / \mathrm{mL}$ or $1000 \mu \mathrm{g} / \mathrm{mL}$ of chitin solubilized in $0.02 \%$ tween 20 . The experiment was repeated four times. This chitin contains 2 to 8-mers of oligosaccharide (YSK, Yaizu Suisankagaku Industry). The third, last fully expanded leaves were harvested, frozen in liquid nitrogen, at different time points after treatment for gene expression quantifications.

\section{$\mathrm{H}_{2} \mathrm{O}_{2}$ measurements and gene expression analysis}

For $\mathrm{H}_{2} \mathrm{O}_{2}$ measurements, leaf fragments were treated as in Vergne et al. [37] using DAB staining. Diaminobenzidine (Sigma D-8001) was solubilized to $1 \mathrm{mg} / \mathrm{ml}$ of water, excised leaves were dipped overnight (in the dark) and tissues were cleared withethanol/chloroform $(4: 1)$ overnight at room temperature. For quantitative RT-PCR applications, frozen tissue were ground in liquid nitrogen. Sampling was done in the growth chambers (with low light in the inoculation dark-room during the first $24 \mathrm{~h}$ ). Each replicate was made from at least 4 plants of one given genotype that were pooled and this design was repeated three to four times. Approximately $500 \mu \mathrm{l}$ of powder was then treated with $1 \mathrm{~mL}$ of TRIZOL (Invitrogen) as recommended. After a DNAse treatment (Euromedex), RNA samples $(5 \mu \mathrm{g})$ were denaturated for $5 \mathrm{~min}$ at $65{ }^{\circ} \mathrm{C}$ with oligo $\mathrm{dT}_{18}(3.5 \mu \mathrm{M})$ and dNTP $(1.5 \mu \mathrm{M})$. They were then subjected to reverse transcription for $60 \mathrm{~min}$ at $37{ }^{\circ} \mathrm{C}$ with $200 \mathrm{U}$ of reverse transcriptase M-MLV (Promega, Madison, WI, USA) in the appropriate buffer. Two microlitres of cDNA (dilution 1/ 10) were then used for quantitative RT-PCR. Quantitative RT-PCR mixtures contained PCR buffer, dNTP (0.25 mM), 
$\mathrm{MgCl}_{2}$ (2.5 mM), forward and reverse primers (150, 300 or $600 \mathrm{nM}$ final concentration), $1 \mathrm{U}$ of HotGoldStar polymerase and SYBR Green PCR mix as per the manufacturer's recommendations (Eurogentec, Seraing, Belgium). Amplification was performed as follows: $95^{\circ} \mathrm{C}$ for $10 \mathrm{~min} ; 40$ cycles of $95^{\circ} \mathrm{C}$ for $15 \mathrm{~s}, 62{ }^{\circ} \mathrm{C}$ for $1 \mathrm{~min}$ and $72{ }^{\circ} \mathrm{C}$ for $30 \mathrm{~s}$; then $95{ }^{\circ} \mathrm{C}$ for $1 \mathrm{~min}$ and $55{ }^{\circ} \mathrm{C}$ for $30 \mathrm{~s}$. The quantitative RT$\mathrm{PCR}$ reactions were performed using a MX3000P machine (Stratagene) and data were extracted using the MX3000P software. The amount of plant RNA in each sample was normalized using actin (Os03g50890) as internal control. Gene expression was done using the measured efficiency for each gene as described in Vergne et al. [37]. The list of primers used is provided in Additional file 6.

\section{Additional files}

Additional file 1: Rice WAKs regulated by blast fungus infection. (PDF $35 \mathrm{~kb}$ )

\section{Additional file 2: Phylogenetic tree of the Arabidopsis WAK and} EGF-containing OsWAK genes from rice. The proteomes of Arabidopsis thaliana (TAIR release 9: 33,200 sequences) and Oryza sativa (TIGR Release 6.0: 67,393 sequences) were downloaded from the GreenPhyl database (http://www.greenphyl.org/cgi-bin/index.cgi) (Conte et al., 2008). We retrieved OSWAK genes proceeding into three steps. First, we ran the hmmsearch program (Eddy, 2009) to search for kinase Hidden Markov Model (HMM) profile (PF00069.16) (Sonnhammer et al., 1998) into Arabidopsis and Oryza sequences. We retrieved 3185 proteins containing a kinase motif. On this set of sequences, we again used the hmmsearch program seeking this time EGFs HMM profiles (PF00008.18, PF09120.1, PF07974.4, PF04863.4 and PF07645.6). From this second screen, we retrieved 248 proteins (33 from Arabidopsis thaliana and 215 from Oryza sativa). We extracted the kinase domain sequences of these proteins and aligned them with the E-INS-i program (default parameters) from the MAFFT website (http://mafft.cbrc.jp/alignment/ software/). Based on this alignment, we generated a phylogenetic tree by the maximum likelihood method with 100 bootstrap replicates. All the genes with a WAK signature, explicitly

containing both EGF motif(s) and kinase domain, were grouped in the tree with a bootstrap value of 86 . All other genes outside this clade have been considered as outgroup. All manipulations on phylogenetic trees have been performed with the treedyn program (http://www.treedyn.org/). Empty circles: newly annotated OsWAK genes; black squares: OSWAK genes known to be differentially expressed upon infection; empty squares: WAK genes known to be involved in fungal resistance; asterics: OsWAK genes with an ACF kinase domain. (PDF $59 \mathrm{~kb}$ )

Additional file 3: Wak insertion mutants. A. For each OsWAK gene, the different splicing forms are shown as well as the position of the T-DNA insertion site. The small arrows designate the primers (Additional file 6) that were used to genotype the plants and the primers used for measuring gene expression by quantitative RT-PCR. B. Transcript levels for the corresponding gene as measured by quantitative RT-PCR in mutant plants (MUT) compared to the corresponding null-segregant (WT) plants. Gene expression was normalized using actin. The values represent the percent of gene expression as compared to WT (100\%); the values are the mean and standard deviation calculated from three biological replicates. (PDF $97 \mathrm{~kb}$ )

Additional file 4: Disease symptoms in T0 transgenic plants over-expressing OsWAK91 or OsWAK112d. Unique rice T0 plants over-expressing OsWAK91 (A, B, C; 5 lines starting with "OE") or OsWAK112d (D, E, F; 6 lines starting with "OX") were produced. Plants transformed with the empty vector are also shown $(A ; 4$ lines starting with " $\left.\mathrm{VV}^{\prime \prime}\right)$. The transgene expression level was normalized using Actin and is expressed as Arbitrary Unit (A, C). The values presented are unique values since each represents a unique T0 plant. Given the extremely low expression levels and thus variability in empty vectors, the very high values in over-expressor lines was considered as significant. Plants were inoculated with the virulent isolate FR13 of Magnaporthe oryzae and lesion number was quantified $7 \mathrm{dpi}(\mathrm{B}, \mathrm{E})$. Examples of symptoms are also shown (C, F). The experiments in panels $A / B$ and $C / D$ have not been conducted at the same time but in two separate experiments. The difference between empty vectors can thus be attributed to differences in the inoculum pressure (higher in the case of A/B). (PDF $2113 \mathrm{~kb}$ )

Additional file 5: Fungal growth in OE-WAK91 lines. WAK91 over-expresser lines were inoculated with M. oryzae (GY11 moderately virulent isolate) and at the indicated time points after inoculation, the development stage of the fungus was observed under the microscope; four categories of growth stages were counted (100 interaction sites/condition). This experiment was repeated 3 times and significant differences (t-test; $P<0.05$ ) are shown by *. (PDF $104 \mathrm{~kb}$ )

Additional file 6: Primers used for this study. (PDF $66 \mathrm{~kb}$ )

\section{Abbreviations}

DAMP: Damage Associated Molecular Pattern; EGF: Epidermal Growth Factor; MAPK: mitogen-activated protein kinases; OG: oligogalacturonide; PAMP: Pathogen Associated Molecular Pattern; PRR: pattern-recognition receptor; PTI: PAMP-triggered immunity; RLK: Receptor-like Kinase; ROS: reactive oxygen species; WAK: Wall-associated kinase.

\section{Competing interests}

The authors declare having no competing interest.

\section{Authors' contributions}

A. Delteil: expression, mutant production and phenotyping, data analysis; EG: expression data; BC: expression, ROS data; JE: mutant analysis, expression; CM; mutant production; A Dievart: phylogenetic analysis; TK: data analysis; JBM: experimental design, data analysis. All authors read and approved the final version of the manuscript.

\section{Acknowledgements}

We are thankful to Loïc Fontaine for taking care of the plants and to Delphine Mieulet for providing mutant seeds from the OryzaTagLine library. AD's work was supported by a PhD grant from CIRAD and Région Languedoc-Roussillon. EG was supported by a INRA post-doc fellowship from INRA-BAP division. This work was supported by the IRMA grant (ANR-07-GPLA-007) from the Génoplante program and the Cerealdefense grant (ANR-09-GENM-106) from the ANR-Génomique program.

\section{Author details}

${ }^{1}$ CIRAD, UMR BGPI INRA/CIRAD/SupAgro, Campus International de Baillarguet, TA A 54/K, 34398 Montpellier, France. ${ }^{2}$ INRA, UMR BGPI INRA/ CIRAD/SupAgro, Campus International de Baillarguet, TA A 54/K, 34398 Montpellier, France. ${ }^{3}$ CIRAD, UMR DAP INRA/CIRAD/SupAgro, Avenue Agropolis, 34398 Montpellier Cedex 5, France.

Received: 16 September 2015 Accepted: 11 January 2016

Published online: 16 January 2016

\section{References}

1. Macho AP, Zipfel C. Plant PRRs and the activation of innate immune signaling. Mol Cell. 2014;54(2):263-72.

2. Boller T, Felix G. A renaissance of elicitors: perception of microbe-associated molecular patterns and danger signals by pattern-recognition receptors. Annu Rev Plant Biol. 2009;60:379-406.

3. Zipfel C. Plant pattern-recognition receptors. Trends Immunol. 2014;35(7):345-51.

4. Shiu SH, Karlowski WM, Pan RS, Tzeng YH, Mayer KFX, Li WH. Comparative analysis of the receptor-like kinase family in Arabidopsis and rice. Plant Cell. 2004;16:1220-34.

5. Kaku H, Nishizawa Y, Ishii-Minami N, Akimoto-Tomiyama C, Dohmae N, Takio K, et al. Plant cells recognize chitin fragments for defense signaling through a plasma membrane receptor. Proc Natl Acad Sci U S A. 2006;103:11086-91. 
6. Gómez-Gómez L, Boller T. FLS2: An LRR Receptor-like Kinase Involved in the Perception of the Bacterial Elicitor Flagellin in Arabidopsis. Mol Cell. 2000;5:1003-11.

7. Miya A, Albert $P$, Shinya $T$, Desaki $Y$, Ichimura $K$, Shirasu $K$, et al. CERK1, a LysM receptor kinase, is essential for chitin elicitor signaling in Arabidopsis. Proc Natl Acad Sci U S A. 2007;104:19613-8.

8. Shimizu T, Nakano T, Takamizawa D, Desaki Y, Ishii-Minami N, Nishizawa $Y$, et al. Two LysM receptor molecules, CEBiP and OsCERK1, cooperatively regulate chitin elicitor signaling in rice. Plant J. 2010;64:204-14.

9. Brutus A, Sicilia F, Macone A, Cervone F, De Lorenzo G. A domain swap approach reveals a role of the plant wall-associated kinase 1 (WAK1) as a receptor of oligogalacturonides. Proc Natl Acad Sci U S A. 2010;107:9452-7.

10. Shibuya N, Nakane R. Pectic polysaccharides of rice endosperm cell walls. Phytochemistry. 1984;23:1425-9.

11. Hynes NE, MacDonald G. ErbB receptors and signaling pathways in cancer Curr Opin Cell Biol. 2009;21:177-84.

12. Schlessinger J. Ligand-induced, receptor-mediated dimerization and activation of EGF receptor. Cell. 2002;110:669-72.

13. He Z-H, Cheeseman I, He D, Kohorn B. A cluster of five cell wall-associated receptor kinase genes, Wak1-5, are expressed in specific organs of Arabidopsis. Plant Mol Biol. 1999;39:1189-96.

14. Verica JA, He ZH. The cell wall-associated kinase (WAK) and WAK-like kinase gene family. Plant Physiol. 2002;129:455-9.

15. Zhang S, Chen C, Li L, Meng L, Singh J, Jiang N, et al. Evolutionary expansion, gene structure, and expression of the rice wall-associated kinase gene family. Plant Physiol. 2005;139:1107-24.

16. Kohorn BD, Kohorn SL, Saba NJ, Martinez VM. Requirement for pectin methyl esterase and preference for fragmented over native pectins for wall-associated kinase-activated, EDS1/PAD4-dependent stress response in Arabidopsis. J Biol Chem. 2014;289(27):18978-86.

17. Li H, Zhou SY, Zhao WS, Su SC, Peng YL. A novel wall-associated receptor-like protein kinase gene, OsWAK1, plays important roles in rice blast disease resistance. Plant Mol Biol. 2009;69:337-46.

18. He ZH, He DZ, Kohorn BD. Requirement for the induced expression of a cell wall associated receptor kinase for survival during the pathogen response. Plant J. 1998;14:55-63.

19. Wang N, Huang HJ, Ren ST, Li JJ, Sun Y, Sun DY, et al. The rice wall-associated receptor-like kinase gene OSDEES1 plays a role in female gametophyte development. Plant Physiol. 2012;160(2):696-707.

20. Zuo W, Chao Q, Zhang N, Ye J, Tan G, Li B, et al. A maize wall-associated kinase confers quantitative resistance to head smut. Nat Genet. 2015;47:151-7.

21. Park AR, Cho SK, Yun UJ, Jin MY, Lee SH, Sachetto-Martins G, et al. Interaction of the arabidopsis receptor protein kinase Wak1 with a glycine-rich protein, AtGRP-3. J Biol Chem. 2001;276:26688-93.

22. Böhm H, Albert I, Fan L, Reinhard A, Nürnberger T. Immune receptor complexes at the plant cell surface. Curr Opin Plant Biol. 2014;20:47-54.

23. Cabrera JC, Boland A, Messiaen J, Cambier P, Van Cutsem P. Egg box conformation of oligogalacturonides: The time-dependent stabilization of the elicitor-active conformation increases its biological activity. Glycobiology. 2008;18:473-82.

24. Decreux A, Thomas A, Spies B, Brasseur R, Van Cutsem P, Messiaen J. In vitro characterization of the homogalacturonan-binding domain of the wall-associated kinase WAK1 using site-directed mutagenesis. Phytochemistry. 2006:67:1068-79.

25. Kohorn BD, Johansen S, Shishido A, Todorova T, Martinez R, Defeo E, et al. Pectin activation of MAP kinase and gene expression is WAK2 dependent. Plant J. 2009;60(2):974-82.

26. Kohorn BD, Kohorn SL, Todorova T, Baptiste G, Stansky K, McCullough M. A dominant allele of Arabidopsis pectin-binding wall-associated kinase induces a stress response suppressed by MPK6 but not MPK3 mutations. Mol Plant. 2012;5(4):841-51.

27. Kohorn BD, Kohorn SL. The cell wall-associated kinases, WAKs, as pectin receptors. Front Plant Sci. 2012;3:88.

28. Wagner TA, Kohorn BD. Wall-associated kinases are expressed throughout plant development and are required for cell expansion. Plant Cell. 2001;13:303-18

29. Kanneganti V, Gupta AK. 2011. RNAi mediated silencing of a wall associated kinase, OsiWAK1 in Oryza sativa results in impaired root development and sterility due to anther indehiscence: Wall Associated Kinases from Oryza sativa. Physiol Mol Biol Plant. 2011;17(1):65-77.
30. Diener AC, Ausubel FM. RESISTANCE TO FUSARIUM OXYSPORUM 1, a dominant Arabidopsis disease-resistance gene, is not race specific. Genetics. 2005;171(1):305-21.

31. Häffner E, Karlovsky P, Splivallo R, Traczewska A, Diederichsen E. ERECTA salicylic acid, abscisic acid, and jasmonic acid modulate quantitative disease resistance of Arabidopsis thaliana to Verticillium longisporum. BMC Plant Biol. 2014;14:85.

32. Hurni S, Scheuermann D, Krattinger SG, Kessel B, Wicker T, Herren G, et al. The maize disease resistance gene $\mathrm{Htn} 1$ against northern corn leaf blight encodes a wall-associated receptor-like kinase. Proc Natl Acad Sci U S A. 2015:112(28):8780-5.

33. Rosli HG, Zheng Y, Pombo MA, Zhong S, Bombarely A, Fei Z, et al. Transcriptomics-based screen for genes induced by flagellin and repressed by pathogen effectors identifies a cell wall-associated kinase involved in plant immunity. Genome Biol. 2013;14(12):R139.

34. Seo YS, Chern M, Bartley LE, Han M, Jung KH, Lee I, et al. Towards establishment of a rice stress response interactome. PLoS Genet. 2011;7: e1002020.

35. Weiberg A, Wang M, Lin FM, Zhao H, Zhang Z, Kaloshian I, et al. Fungal small RNAs suppress plant immunity by hijacking host RNA interference pathways. Science. 2013;342(6154):118-23.

36. Bagnaresi $P$, Biselli $C$, Orrù L, Urso $S$, Crispino L, Abbruscato $P$, et al. Comparative transcriptome profiling of the early response to Magnaporthe oryzae in durable resistant vs susceptible rice (Oryza sativa L.) genotypes. PLoS One. 2012;7:e51609.

37. Vergne E, Ballini E, Marques S, Mammar BS, Droc G, Gaillard S, et al. Early and specific gene expression triggered by rice resistance gene Pi33 in response to infection by ACE1 avirulent blast fungus. New Phytologist. 2007; 174:159-71.

38. Denoux C, Galletti R, Mammarella N, Gopalan S, Werck D, De Lorenzo G, et al. Activation of defense response pathways by OGs and Flg22 elicitors in Arabidopsis seedlings. Mol Plant. 2008;1(3):423-45.

39. Zipfel C, Robatzek S, Navarro L, Oakeley EJ, Jones JDG, Felix G, et al. Bacteria disease resistance in Arabidopsis through flagellin perception. Nature. 2004; 428:764-7.

40. Meier S, Ruzvidzo O, Morse M, Donaldson L, Kwezi L, Gehring C. The Arabidopsis wall associated kinase-like 10 gene encodes a functional guanylyl cyclase and is co-expressed with pathogen defense related genes. PLoS One. 2010;5:e8904.

41. Vander P, Varum KM, Domard A, Eddine El Gueddari N, Moerschbacher BM. Comparison of the ability of partially $\mathrm{N}$-acetylated chitosans and chitooligosaccharides to elicit resistance reactions in wheat leaves. Plant Physiol. 1998;118:1353-9.

42. Kishimoto K, Kouzai Y, Kaku H, Shibuya N, Minami E, Nishizawa Y. Perception of the chitin oligosaccharides contributes to disease resistance to blast fungus Magnaporthe oryzae in rice. Plant J. 2010;64:343-54

43. Kouzai Y, Nakajima K, Hayafune M, Ozawa K, Kaku H, Shibuya N, et al. CEBiP is the major chitin oligomer-binding protein in rice and plays a main role in the perception of chitin oligomers. Plant Mol Biol. 2014;84(4-5):519-28.

44. Delteil A, Blein M, Faivre-Rampant O, Guellim A, Estevan J, Hirsch J, et al. Building a mutant resource for the study of disease resistance in rice reveals the pivotal role of several genes involved in defense. Mol Plant Pathol. 2012; 13(1):72-82.

45. Larmande P, Gay C, Lorieux M, Perin C, Bouniol M, Droc G, et al. Oryza Tag Line, a phenotypic mutant database for the Genoplante rice insertion line library. Nucleic Acids Res. 2008;36:1022-7.

46. Sallaud C, Gay C, Larmande P, Bès M, Piffanelli P, Piégu B, et al. High throughput T-DNA insertion mutagenesis in rice: a first step towards in silico reverse genetics. Plant J. 2004;39:450-64.

47. Liu B, Li JF, Ao Y, Qu J, Li Z, Su J, et al. Lysin motif-containing proteins LYP4 and LYP6 play dual roles in peptidoglycan and chitin perception in rice innate immunity. Plant Cell. 2012;8:3406-19.

48. Keinath NF, Kierszniowska S, Lorek J, Bourdais G, Kessler SA, Asano H, et al. PAMP-induced changes in plasma membrane compartmentalization reveal novel components of plant immunity. J Biol Chem. 2010;285(50):39140-9.

49. Dardick $C$, Ronald P. Plant and animal pathogen recognition receptors signal through non-RD kinases. PLoS Pathog. 2006;2(1):e2.

50. Berruyer R, Adreit H, Milazzo J, Gaillard S, Berger A, Dioh W, et al. Identification and fine mapping of Pi33, the rice resistance gene corresponding to the Magnaporthe grisea avirulence gene ACE1. Theor Appl Genet. 2003;107:1139-47. 